\title{
Comparison of the Accuracy of MRI, Sonography and Mammography in Predicting the Pre Operative Breast Tumor Size Measurements
}

\author{
Authors \\ Dr Mohammed Ameer Ali Khan Afrose', Dr Yuefan Gu², Dr Junkang Shen, \\ Dr Uzma Jabeen ${ }^{4}$ \\ ${ }^{1,2}$ MBBS (MD Radiology), Second affiliated hospital of Soochow University \\ ${ }^{3}$ HOD, MRI Department, Soochow University The Second Affiliated Hospital of Scoochow University \\ Institute of Radiotherapy \&Oncology Scoochow University Suzhou, 215004 China, \\ ${ }^{4}$ Shadan Medical College
}

\section{ABSTRACT}

Background: 70 patients with primary breast cancer who presented preoperatively in the New Life Hospital, Hyderabad from Jan 2016 to June 2016 were retrospectively analyzed. Accurate preoperative assessment of maximum tumor size was a significant step of clinical cancer staging that assisted in planning further patient managements. The commonly used radiological modalities to measure tumor size are mammography, Ultra Sonography (US), and MRI. To prospectively estimate the accuracy of mammography, Ultrasonography and Magnetic Resonance Imaging in preoperative assessment of local extent of breast cancer, we did a study which illustrated the purpose. Bilateral whole-breast Ultrasonography was performed with prior information about clinical and mammographic findings either prior to MR imaging or afterward.

Methods: Data from 70 patients with primary breast cancer were analysed in a retrospective study. The results were divided into the groups "ductal carcinoma in situ (DCIS)", invasive ductal carcinoma (IDC) + ductal carcinoma in situ (DCIS)", "invasive ductal carcinoma (IDC)", "invasive lobular carcinoma (ILC)" and "other tumours" (tubular, medullary, mucinous and papillary breast cancer). The largest tumour diameter was chosen as the sizing reference in each case. Data from the study was diagrammatically illustrated and study data was incorporated in table form to accurately differentiate between the modalities so as to come to conclusions.

Result: Our study found that ultrasound produced significant underestimation of tumor size, particularly so when size of tumor is larger. Mammography also under estimates the size of the tumor as compared to histology. MRI gives most accurate size estimation. However, overall mean difference between mammography, Ultrasonography and MRI was not statistically significant.

Conclusion: We state that all 3 modalities of imaging has a role in pre operative estimation of breast tumor. As depicted in our study, MRI has shown most accurate results.

Abbreviations: DCIS: Ductal Carcinoma in Situ, IDC: Invasive Ductal carcinoma, ILC: Invasive Lobular carcinoma

Keywords: Breast tumour, BI RADS classification, Ultra sonography, mammography, MRI. 


\section{Introduction}

The breast is the most common site of cancer occurring in women and carcinoma of the breast is second to lung carcinoma as a cause of death from cancer among women.(1) Though, they may arise from various sources - connective tissue or epithelial structures, latter gives rise to most common breast carcinoma. Breast Carcinoma is the most common cancer in females with the ratio between male to female being 1:200 . Breast cancer seems to be due to a constellation of epidemiologic factors rather than to a single one, including genetic predisposition (BRCA1 \{chromosome 17\} with BRCA2 \{chromosome $13\}$ ), carcinogen exposure with various adverse personal with demographic conditions; Hence, it would seem highly improbable that an epidemiological factor of overwhelming importance in breast cancer will be found. ${ }^{(2)}$ Even though there are few preventive measures that may be of importance, most of the causative factors in breast cancer are beyond the imaginations of physicians and patients, so the best way to reduce the consequences of carcinoma of the breast is early and right diagnosis and staging with treatment according to it, at the earliest moment possible. Therefore, prognosis of carcinoma of the breast seems to be based on the dynamic interaction between the anatomic extent of cancer, its early diagnosis with its growth potential, i.e. aggressiveness or virulence or invasiveness, on one side the degree of immune competence of the host with appropriate early treatment on the other side. $(3,4)$ The treatment and management of carcinoma of the breast depends upon the stage at the time of presentation of patient, which depends on the involvement of lymphatics, neighboring tissue with distant metastasis.

\section{Imaging modalities, method and equipment used}

Accurate preoperative assessment of maximum tumor size is a significant step of clinical cancer staging that assist in planning further patient management. Though histopathologic staging remains the gold standard, management decisions are frequently made preoperatively on the basis of clinical tumor size assessment on physical examination and imaging. Preoperatively, tumor size is taken for clinical staging and assessing patients' condition for breast conservation and neoadjuvant chemotherapy. The commonly used radiological modalities to measure tumor size are mammography, sonography (US), and MRI.

To prospectively estimate the accuracy of mammography, clinical examination, ultrasonography and magnetic resonance imaging in preoperative assessment of local extent of breast cancer, we have done a study which illustrates the purpose.

Mammography forms the gold standard for breast cancer screening; ${ }^{(5,6)}$ But for women with increased risk of breast cancer, other screening modalities, like Ultrasonography and MRI, have shown to contribute to early the detection of breast cancer. MRI of the breast is commonly used now days to screen for breast cancer in the high-risk patient.

Bilateral mammography is performed which includes routine craniocaudal and mediolateral oblique views of the breast and spot- or globalmagnification views over the area of the cancer. Findings of mammography are recorded prospectively according to the Breast Imaging Reporting and Data System (BI-RADS lexicon). Findings which are mammographically probably benign, suspicious for cancer, or highly suggestive of malignancy, are sampled for biopsy, if the patient is a candidate for conservation and if identification of a malignancy at that site would alter the surgical approach.

Bilateral whole-breast Ultrasonography is performed with prior information about of clinical and mammographic findings either prior to MR imaging or afterward. By using a linear-array broadband transducer with a center frequency of $10 \mathrm{MHz}$, Ultrasonography is performed and supplemented with a linear-array transducer along with a center frequency of $7.5 \mathrm{MHz}$ as required 
for penetration of larger breasts (Acoustic Imaging Performa, Tempe, Ariz, or Elegra, Siemens, Issaquah, Ish). For the inner breast, scanning is done with the patient in the supine posture. For the outer breast, the patient is positioned in the contra lateral posterior oblique position with the ipsilateral arm raised. The examination is carried out using "Spatial Compound Imaging" and "XRES Adaptive Image Processing". Measurement of tumor size takes the echo poor centre of the lesion and the echogenic halo into account. Survey scanning is done in transverse and sagittal planes. Discrete lesions are estimated in both radial and anti radial scanning planes. When multiple suspicious lesions are found, panoramic display is used as and when possible to document the distance between lesions.

Images and perpendicular measurements are noted for most of discrete findings other than simple cysts. Biopsy is advised for all palpable solid masses and for incidental solid masses unless they are (a) circumscribed, oval, uniformly hypoechoic with no posterior features, and non palpable or $(b)$ non palpable complicated cysts. The latter two classes of lesions are classified as probably benign at utrasonography and are recommended for short-interval (6-month) follow-up. If such lesions match to suspicious findings at $\mathrm{MR}$ imaging, then biopsy is performed, but the ultrasonography classification remained probably benign for purposes of analysis.

MR imaging is done with the patient in the prone position in a dedicated phased-array breast coil. Transverse T1-weighted MR images (repetition time msec/echo time msec, 718/14; two signals acquired; field of view, $32-40 \mathrm{~cm}$; section thickness, $5 \mathrm{~mm}$ ) are obtained in both breasts, followed by sagittal T2-weighted fat-suppressed.

\section{Material \& Methods}

70 patients with primary breast cancer who presented preoperatively in the New Life Hospital, Hyderabad from Jan 2016 to June 2016 were retrospectively analysed.

\section{Inclusion Criteria}

$>$ Primary breast cancer

$>$ No neoadjuvant chemotherapy

$>$ Documentation of the tumor size from mammography

$>$ Sonography and Magnetic resonance imaging (MRI) as well as the postoperative histological tumor size. (largest tumor diameter was taken as the sizing reference in each case ).

\section{Exclusion Criteria}

$>$ Histological size was not measurable

$>$ Only in situ disease was present

$>$ Chemotherapy or Neo adjuvant chemotherapy.

$>$ Multifocal tumor

$>$ Tumor was not seen on mammography/ ultrasound

\section{Statistical Evaluation}

The histological results were treated with relevant statistic method. The largest tumor diameter was chosen as the sizing reference in each case. The mean difference between the imaging and the histological results was calculated and related to the interval in which $95 \%$ of the calculated differences were found. Size variation on imaging versus pathology was reported as median and interquartile range, using Box plots. The statistical analysis was performed using MINITAB version 17. The level of significance was defined as a $\mathrm{p}$ value of $<0.05$. 
]Results

Diagram 1. Correlation between BI RADS and Histology

\begin{tabular}{|l|c|c|c|c|c|c|c|}
\hline STUDY GROUPS & $\begin{array}{c}\text { BI RADS } \\
\text { CLASSIFICATION }\end{array}$ & $\begin{array}{c}\text { ISOLATED } \\
\text { DCIS }\end{array}$ & $\begin{array}{c}\text { IDC - } \\
\text { DCIS }\end{array}$ & $\begin{array}{c}\text { ISOLATED } \\
\text { IDC }\end{array}$ & $\begin{array}{c}\text { ISOLATED } \\
\text { ILC }\end{array}$ & $\begin{array}{c}\text { OTHER } \\
\text { TUMOR }\end{array}$ & TOTAL \\
\hline MAMMOGRAPHY & 0 & 0 & 0 & 0 & 1 & 0 & 1 \\
\hline & 1 & 0 & 0 & 1 & 0 & 0 & 1 \\
\hline & 2 & 0 & 0 & 0 & 0 & 0 & 0 \\
\hline & 3 & 0 & 1 & 2 & 1 & 1 & 5 \\
\hline & 4 & 2 & 8 & 9 & 5 & 5 & 29 \\
\hline & 5 & 7 & 9 & 10 & 5 & 3 & 34 \\
\hline SLTRA & 6 & 0 & 0 & 0 & 0 & 0 & 0 \\
\hline & 0 & 0 & 0 & 0 & 0 & 0 & 0 \\
\hline & & 0 & 0 & 0 & 0 & 0 & 0 \\
\hline & 2 & 0 & 0 & 0 & 1 & 0 & 1 \\
\hline & 3 & 0 & 2 & 0 & 0 & 0 & 2 \\
\hline & 4 & 6 & 8 & 6 & 4 & 2 & 30 \\
\hline MRI & 5 & 4 & 9 & 12 & 5 & 1 & 36 \\
\hline & 6 & 0 & 0 & 0 & 0 & 0 & 0 \\
\hline & 0 & 0 & 0 & 0 & 0 & 0 & 0 \\
\hline & 1 & 0 & 0 & 0 & 0 & 0 & 0 \\
\hline & 2 & 0 & 0 & 0 & 0 & 0 & 0 \\
\hline & 3 & 0 & 0 & 0 & 0 & 0 & 0 \\
\hline & 4 & 0 & 1 & 0 & 0 & 0 & 1 \\
\hline & 5 & 5 & 10 & 13 & 3 & 1 & 32 \\
\hline
\end{tabular}

DCIS: Ductal Carcinoma in Situ, IDC : Invasive Ductal carcinoma, ILC : Invasive Lobular carcinoma

Diagram 2. Comparison of Imaging size and histological tumor size

\begin{tabular}{|l|c|c|c|c|c|c|c|c|c|}
\hline \multirow{2}{*}{ TUMOR GROUP } & \multicolumn{3}{|c|}{$\begin{array}{c}\text { MAMMOGRAPHY AND } \\
\text { HISTOLOGY }\end{array}$} & \multicolumn{2}{c|}{ SONOGRAPHY AND HISTOLOGY } & \multicolumn{3}{c|}{ MRI AND HISTOLOGY } \\
\cline { 2 - 10 } & $\mathrm{M}(\mathrm{)}$ & $\begin{array}{c}\text { LOA( } \\
\mathrm{mm})\end{array}$ & $\mathrm{r}$ & $\mathrm{M}(\mathrm{mm})$ & $\mathrm{LOA}(\mathrm{mm})$ & $\mathrm{r}$ & $\mathrm{M}(\mathrm{mm})$ & $\begin{array}{c}\text { LOA( } \\
\mathrm{mm})\end{array}$ & $\mathrm{r}$ \\
\hline DCIS & 2 & -67 to 58 & 0.42 & -12 & -76 to 52 & 0.43 & 4 & -43 to 58 & 0.67 \\
\hline IDC-DCIS & -3 & -39 to 27 & $\begin{array}{c}0 . \\
35\end{array}$ & -7 & -52 to 34 & 0.58 & 3 & -40 to 52 & 0.42 \\
\hline IDC & 4 & -17 to 24 & 0.65 & -3 & -19 to 11 & 0.76 & 2 & -16 to 28 & 0.64 \\
\hline ILC & 2 & -19 to 17 & 0.76 & -8 & -28 to 10 & 0.52 & -3 & -28 to 37 & 0.72 \\
\hline OTHER TUMORS & -3 & -12 to 16 & 0.82 & -5 & -14 to 32 & 0.88 & 2 & -12 to 14 & 0.81 \\
\hline TOTAL & -2 & -35 to 38 & 0.51 & $\begin{array}{c}-7 \\
(\mathrm{p}>0.005)\end{array}$ & -36 to 20 & 0.49 & $\begin{array}{c}1 \\
(\mathrm{p}>0.005)\end{array}$ & -27 to 42 & 0.48 \\
\hline
\end{tabular}

Diagram 3. Box plot of Median size difference between imaging and Histological size of breast tumors

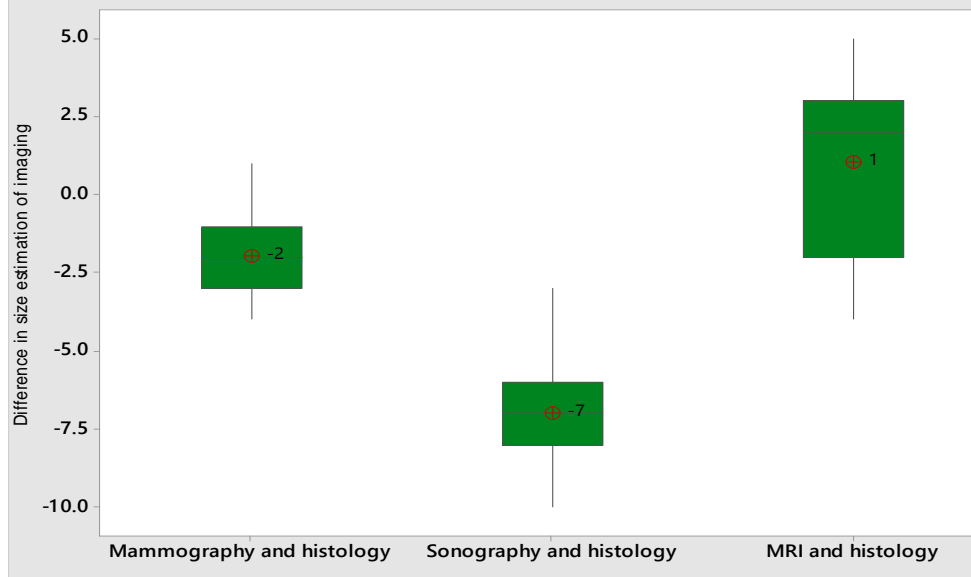




\section{JMSCR Vol||04||Issue||09||Page 12523-12531||September}

Diagram 4 . Individual plot showing size difference between Mammography and Histology

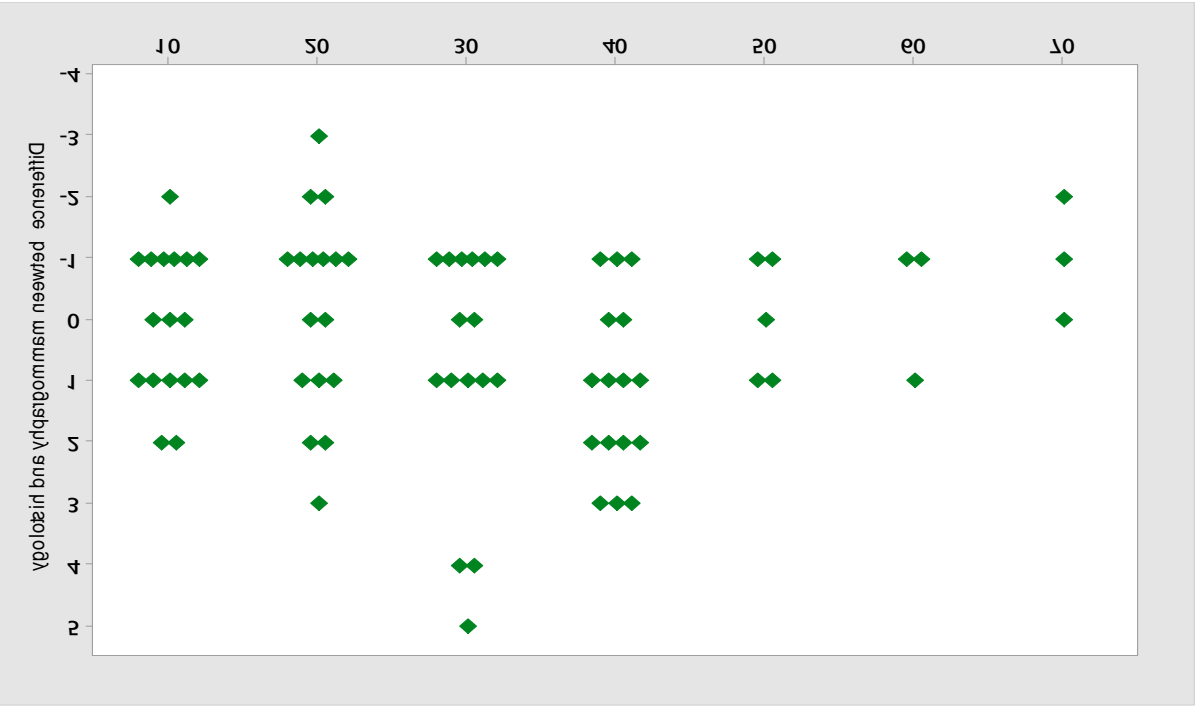

Diagram 5 . Individual plot showing size difference between Ultrasonography and Histology

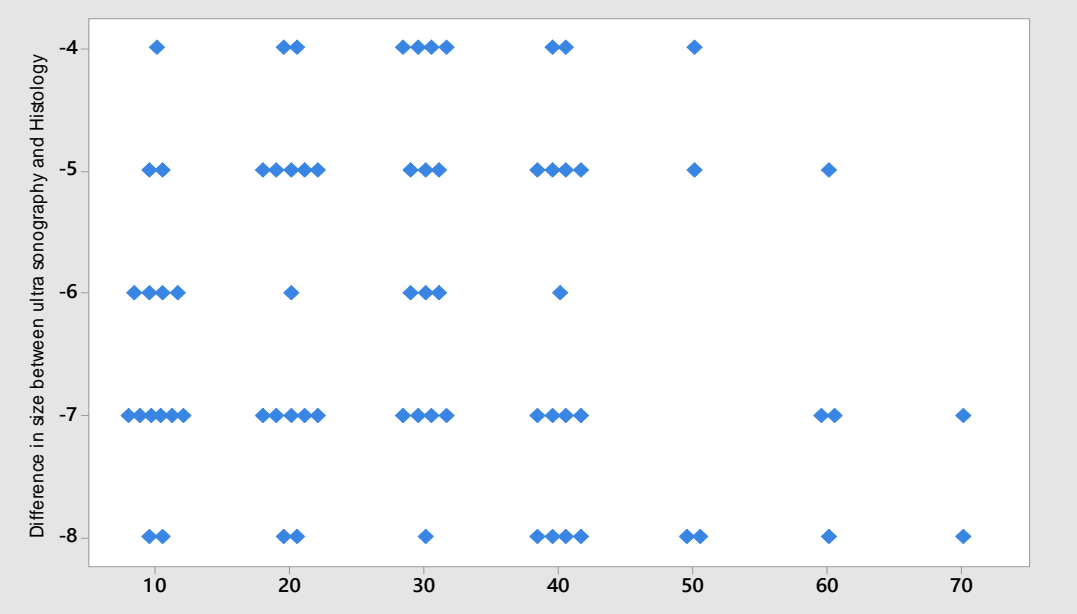

Diagram 6 . Individual plot showing size difference between MRI and Histology

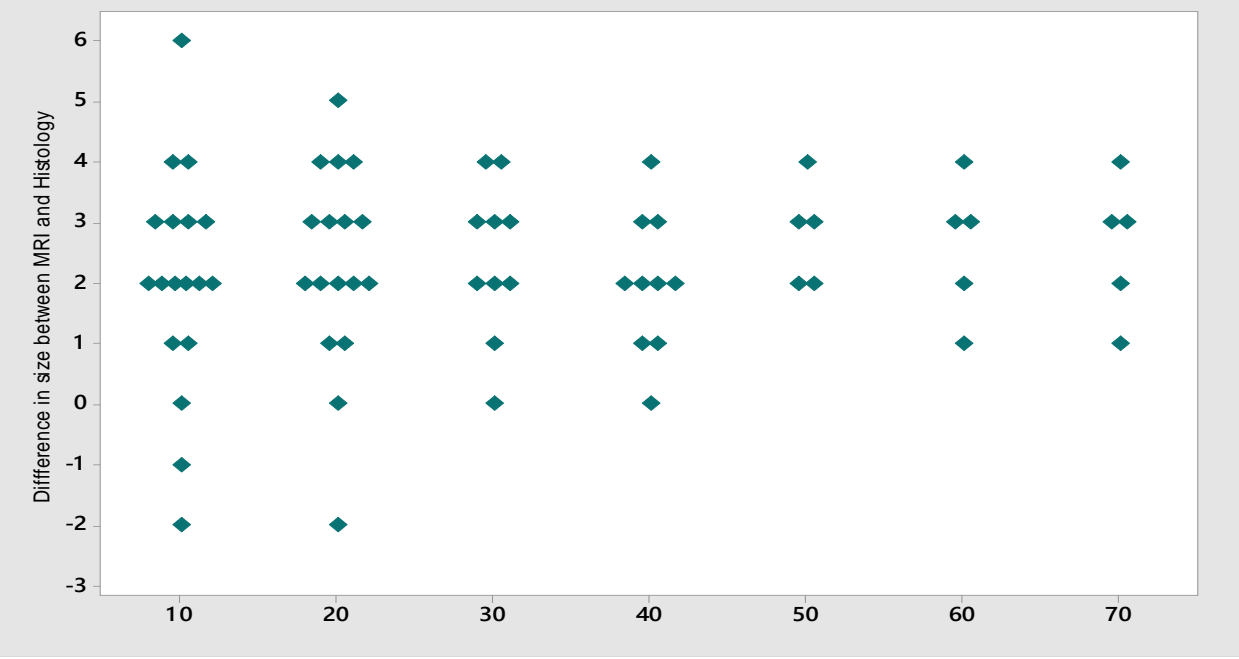


The mean of difference for mammography and histology was -2 and mean of difference for ultrasonography and histology was -7 , and for MRI and histology was 1 . Standard deviation of difference for mammography and histology was 0.48 , for ultrasonography was 0.76 and for MRI and histology was 0.92. The mean size for mammography was $6.08 \mathrm{~cm}$, for ultrasonography $5.43 \mathrm{~cm}$, and for MRI $6.21 \mathrm{~cm}$, and for histopathology $6.12 \mathrm{~cm}$.

The 95\% confidence limit for mammography (mean size \pm 2 standard deviation) was 2.26 $9.4 \mathrm{~cm}$, for ultrasonography $1.23-8.3 \mathrm{~cm}$, for MRI 0.88-7.88 cm, and for histopathology $1.08-8.2$ $\mathrm{cm}$. The z-test value for mammography it was 3.26 , for ultrasonography it was -0.41 and for MRI it was 0.56 .

Analysis shows highly significant underestimation of the mean histological tumour size with sonography $(\mathrm{p}<0.005)$, with a mean of $5.43 \mathrm{~cm}$. This underestimation increased as the histological result size increased. The investigations by Keliska et al., Rekoal et al. had showed the ultra sonographic underestimation of the histological tumor size. Hieken et al. attributed this to the unclear margins of ultra sonographic results from extensive intraductal in-situ components. Bosch et al. reasoned the underestimation with tumor size, with the image presentation more than what is possible with the transducer. However, if panorama mode was used that would have given more accurate estimation of tumor size. With respect statistical method used, if such large tumors were excluded from the study, more accurate tumor size estimation could have been achieved.

Regarding mammographic estimation, our data also show an underestimation of the mean histological result of $0.86 \mathrm{~mm}$. Howver it was not found to be statistically significant. The study by Bikern et al. also emphasized a size underestimation with mammography. Six other studies from the literature found that mammography underestimated size. On the contrary, four other studies found that mammography overestimated size. ${ }^{(7,8)}$ There was also lesser agreement on the nature of the mammographic abnormality. This shows the highly subjective character of these mammographic observations.

MRI results showed size overestimation of $1 \mathrm{~mm}$. Mitashi et al. in their study had found a significant MRI mean overestimation of $1.28 \mathrm{~cm}$. many studies have cooraborated similar results and more so if size of the tumor was $>2 \mathrm{~cm}$ in size.$^{(9,10,11)}$ This can be traced back to tumors with larger DCIS-components or a higher proportion of fibrotic tissue.

Studies which comparatively analysed the diagnostic measurement accuracy of mammography, sonography and MRI came to the conclusion that MRI offered the best correlation with the histological tumor size. For a mean histological tumor size of $3 \mathrm{~cm}$, Rao BK et al. identified a mean tumor site of $2.4 \mathrm{~cm}$ using mammography, $1.9 \mathrm{~cm}$ using sonography and $2.68 \mathrm{~cm}$ with MRI. In a study by Boetes et al. the tumor size with mammography and sonography was underestimated in $14 \%$ and $18 \%$ of the results respectively, whereas MRI did not show any significant deviation from the histological sizing. ${ }^{(13,14,15)}$

\section{Differences in size estimation in different modalities of imaging}

Out study revealed a significant under estimation of the tumor size with ultrasound with regard to the tumor groups IDC-DCIS $(p=0.003)$, IDC $(p=0.004)$ and ILC $(p=0.004)$. The highest mean difference between the ultra sonographically measured tumor size and the actual histological tumor size was found for DCIS, followed by IDCDCIS and ILC. Pritt et al. described the greatest sonographic size underestimation for ILC compared to IDC or ILC-IDC, with a median of $7.5 \mathrm{~mm}$. In our study, we derived a mean size underestimation of $8 \mathrm{~mm}$ in this group. Diagnostic demarcation of the tumor using ultra sonography is made more difficult because of the diffuse, infiltrative growth pattern of ILC. Furthermore, 
ILC may be become multifocality due formation of peritumoral satellite foci. ${ }^{(16,17)}$

Mammography revealed underestimation of tumor size as determined histologically. Few possible sources of error are discussed as a reason. Firstly, there is difficulty in defining accurate margins of the mammographic abnormality. We found that there is a significant tendency to underestimate size of tumor when the mammographic abnormality is classified as a disturbance of breast architecture. The second source of error is radiographic magnification. Pain et al. used tumor model studies to demonstrate a $10 \%$ magnification for a $1 \mathrm{~cm}$ tumor $5 \mathrm{~cm}$ away from the film. Sphiris and Flannagan have both proposed correction factors to compensate for magnification. ${ }^{(18,19,20)}$ It is also possible that none of the standard mammographic projections demonstrated the largest tumor diameter. ${ }^{(21,22)}$

In contrast to mammography and sonography, all tumors were correctly preoperatively classified as requiring further clarification (>BI-RADS IV) with MRI. ${ }^{(23,24)}$ With regard to sizing, there is a non-significant overestimation of size with MRI in all tumor groups. Analogous to our data, other studies show that MRI is superior to both mammography and sonography in the diagnosis of DCIS and ILC. In a study by Kuhl et al. ${ }^{(25,26,27)}$ MRI showed sensitivity for all DCIS cases, whether with or without microcalcification, of $98 \%$.

\section{Study Limitations}

Study was conducted on sample size of 70 . However, as the sample size was small, extrapolation of the results of study to the population is limited. This study was performed by a single radiologist. Hence where ever subjective estimation of size of tumors is involved, findings may be limited to that extent.

\section{Conclusion}

Our study found that ultrasound produced significant underestimation of tumor size, particularly so when size of tumor is larger .Our study found that mammography also under estimates the size tumor as compared to histology. MRI showed most accurate size estimation. However, overall mean difference between mammography, Ultrasonography and MRI was not statistically significant. Various features of the imaging process have been studied. Only the character of the mammographic abnormality, a subjective observation, is shown to influence the accuracy of measurement. However, if the estimation is corroborated with another radiologist's estimation, subjectivity may come down. Thus we state that all 3 modalities of imaging has a role in pre operative estimation of breast tumor. As depicted in our study, MRI has shown most accurate results.

\section{Competing interests}

The authors declare that they have no competing interests

\section{References}

1. Kaufmann M, von Minckwitz G, Smith R, Valero V, Gianni L, Eiermann W, et al. International expert panel on the use of primary (preoperative) systemic treatment of operable breast cancer: review and recommendations. J Clin Oncol 2003; 21:2600-8

2. Buchberger W, DeKoekkoeK Doll P, Springer P, Obrist P, Dunser M. Incidental findings on sonography of the breast: clinical significance and diagnostic workup. AJR Am J Roentgenol. 1999; 173:921-927.

3. Kolb TM, Lichy J, Newhouse JH. Occult cancer in women with dense breasts: detection with screening US - diagnostic yield and tumor characteristics. Radiology. 1998;207:191-199.

4. Schulz KD, Albert US. Stufe-3-Leitlinie Brustkrebs- Früherkennung in Deutschland. München: Zuckschwerdt; 2003.

5. Kolb TM, Lichy J, Newhouse JH. Comparison of the performance of screen- 
ing mammography, physical examination, and breast US and evaluation of factors that influence them: an analysis of 27,825 patients evaluations. Radiology. 2002; 225:165-175.

6. Boyd NF, Guo H, Martin LJ, Sun L, Stone J, Fishell E, Jong RA, Hislop G, Chiarelli A, Minkin S, Yaffe MJ. Mammographic density and the risk and detection of breast cancer. N Engl J Med. 2007;356:227-336.

7. Skaane $P$, Engedal K. Analysis of sonographic features in the differentiation of fibroadenomas and invasive ductal carcinoma. AJR Am J Roentgenol. 1998; 170:109-114

8. Peintinger $\mathrm{F}$, Kuerer $\mathrm{H}$, Anderson $\mathrm{K}$, Boughey J, Meric-Bernstam F, Singletary $S$, et al. Accuracy of the combination of mammography and sonography in predictting tumour response in breast cancer patients after neoadjuvant chemotherapy. Ann Surg Oncol 2006; 13:1443-9

9. Rhabar G, Sie AC, Hansen GC, Prince JS, Melany ML, Reynolds HW, Jackson VP, Syre JW, Bassett LW. Benign versus malignant solid breast masses: US differentiation. Radiology. 1999; 213:889-894.

10. Booser D, Hortobagyi G. Treatment of locally advanced breast cancer. Semin Oncol 1992;19:278-85

11. Mendelson EB, Baum JK, Berg WA, Merritt CRB, Rubin E. Breast Imaging Reporting and Data System, BI-RADS: Ultrasound. Reston: American College of Radiology; 2003.

12. Landis JR, Koch GG. An application of hierarchical kappa-type statistics in the assessment of majority agreement among multiple observers. Biometrics 1977; 33:363-74

13. Baez E, Strathmann K, Vetter M, Madjar $\mathrm{H}$, Hackelöer BJ. Likelihood of malignancy in breast lesions characterized by ultrasound with a combined diagnostic score. Ultrasound Med Biol. 2005;31:179184.

14. Elmore JG, Armstrong K, Lehmann CD, et al. Screening for breast cancer. JAMA. 2005;293:1245-1256.

15. Madjar $H$, Ohlinger R, Mundinger A, Watermann D, Frenz JP, Bader W, SchulzWendtland R, Degenhardt F. BI-RADSanalogue DEGUM criteria for findings in breast ultrasound - consensus of the DEGUM Committee on Breast Ultrasound. Ultraschall Med. 2006;27:374-379.

16. Chen JH, Mehta RS, Nalcioglu O, Su MY. Inflammatory breast cancer after neoadjuvant chemotherapy: can magnetic resonance imaging precisely diagnose the final pathological response? Ann Surg Oncol2008;15:3609-13

17. Moss HA, Britton PD, Flower CD, et al. How reliable is modern breast imaging in differentiating benign from malignant breast lesions in the symptomatic population? Clin Radiol. 1999;54:676-682.

18. Shoma A, Moutamed A, Ameen M, Abdelwahab A: Ultrasound for accurate measurement of invasive breast cancer tumor size. Breast J. 2006, 12: 252-256

19. Wasif N, Garreau J, Terando A, Kirsch D, Mund DF, Giuliano AE: MRI versus ultrasonography and mammography for preoperative assessment of breast cancer. Am Surg. 2009, 75: 970-975.

20. Skaane P, Skjorten F: Ultrasonographic evaluation of invasive lobular carcinoma. Act Radiol. 1999, 40: 369-375.

21. Stomper PC, Margolin FR: Ductal carcinoma in situ: the mammographer's perspective. AJR Am J Roentgenol. 1994, 162: 585-591.

22. Sardanelli F, Giuseppetti GM, Panizza P, Bazzocchi M, Fausto A, Simonetti G, Lattanzio V, Del Maschio A: Sensitivity of MRI versus mammography for detecting foci of multifocal, multicentric breast cancer in fatty and dense breasts using the 
whole breast pathologic examination as a gold standard. AJR Am J Roentgenol. 2004, 183: 1149-1157.

23. Lenz S: Breast ultrasound in office gynecology--ten years of experience. Ultrasc-hall Med. 2011, 32 (Suppl 1): 3-7.

24. Landis JR, Koch GG. An application of hierarchical kappa-type statistics in the assessment of majority agreement among multiple observers. Biometrics. 1977;33 (2):363-374.

25. Buchholz TA, Tucker SL, Masullo L, et al. Predictors of local-regional recurrence after neoadjuvant chemotherapy and mastectomy without radiation. $\mathbf{J}$ Clin Oncol. 2002;20(1):17-23.

26. Golshan M, Fung BB, Wiley E, et al. Prediction of breast cancer size by ultrasound, mammography and core biopsy. The Breast. 2004;13(4):265-271

27. Fornage B, Toubas O, Morel M. Clinical, mammographic, and sonographic determination of preoperative breast cancer size. Cancer. 1987;60:765-771. 